\title{
DEFICIENCIA ECONÓMICA EN GESTIÓN SOCIAL DE LA SOCIEDAD DE BENEFICENCIA PÚBLICA - CALLAO
}

\author{
ECONOMIC DEFICIENCY IN SOCIAL MANAGEMENT OF THE PUBLIC BENEFIT \\ SOCIETY-CALLAO \\ Juan Antonio Arrunategui Aguirre * \\ Unidad de Posgrado de la Facultad de Ciencias Contables \\ Universidad Nacional Mayor de San Marcos-UNMSM / Lima-Perú \\ [Recepción: Febrero 2016/ Conformidad: Mayo 2016]
}

\section{RESUMEN}

La investigación ha proporcionado el conocimiento de las causas de la realidad problemática de la Sociedad de Beneficencia Pública del Callao, en relación a su rentabilidad y liquidez, impidiendo que la entidad pueda cumplir efectivamente con sus metas y objetivos; en consecuencia, creemos que se justifica en la medida, que es necesario, cumplir con la población excluida, grupos de extrema pobreza y vulnerables; sin que ello signifique, el incumplimiento con el normal funcionamiento administrativo y operativo de la institución más antigua del Callao.

\section{Palabras Clave:}

Beneficencia, rentabilidad, liquidez, grupo de extrema pobreza, vulnerables.

\begin{abstract}
The investigation has provided knowledge of the causes of the problematic reality of the Public Benefit Society of Callao, in relation to its profitability and liquidity, preventing the entity of effectively meeting its goals and objectives. In consequence, we believe that its existence is justified to the extent that it is necessary to assist the excluded population in extreme poverty and vulnerable groups; nonetheless this does not mean a non-compliance with the normal administrative and operational functioning of the oldest institution in Callao.
\end{abstract}

\section{Keywords:}

Public charity, profitability, liquidity, group of extreme poverty, vulnerable.

\footnotetext{
*Egresado de la Maestría en Contabilidad en mención en Contabilidad de Gestión. Email: cpcjaugui@hotmail.com
} 


\section{INTRODUCCIÓN}

En el año 1893, siendo presidente del Perú el Coronel Remigio Morales Bermúdez, se dio la primera Ley de Instituciones Benéficas, con el fin de apoyar a las personas de bajos recursos económicos y pobreza extrema.

El 01 de Diciembre de 1848 se fundó la sociedad de beneficencia pública del Callao bajo el gobierno del Presidente Ramón Castilla y su primer Presidente de Directorio fue el Prócer de la Independencia Manuel Cipriano Dulanto Valenzuela, teniendo como misión el brindar apoyo social a los niños, adolescentes, jóvenes, mujeres, ancianos, enfermos y discapacitados de los sectores más pobres, que se encuentra en estado de desamparo familiar y social y su visión, ser una institución moderna, eficiente, oportuna, orientada a brindar refugio social, e integral, propuesta de gestión mediante la aplicación de políticas y planes de desarrollo programadas y de proyección social.

En ese sentido, el presente trabajo de investigación, titulado "EL PROBLEMA DE LIQUIDEZ Y RENTABILIDAD CON EL ENFOQUE SOCIAL EN LA GESTIÓN DE LA SOCIEDAD BENEFICENCIA PÚBLICA DEL CALLAO 2012-2014", tiene como objetivo determinar el factor o factores que causan esta falta de liquidez, y por consiguiente su rentabilidad, lo cual conllevaría a no poder cumplir con sus fines de apoyo social y su gestión administrativa.

Los ingresos que recibe la beneficencia del Callao proviene del alquiler de inmuebles y oficinas (http: //www.sbpcallao.gob.pe/TUPA.Ltml, El Campo Santo Baquijano y Carrillo, en la cual se proporciona los servicios de sepelios funerarios, (http:// baquijano.sbpcallao,gob.pe/) y sus egresos que son fines benéficos a través de campañas cívicas en forma periódica en las comunidades más vulnerables y necesitadas de la región Callao, misma que consiste en llevar alimentos no perecibles, atención médica y entrega de medicinas.

Se mantiene 7 comedores, dando alimento en forma gratuita de lunes a sábado a un total de 1,473 beneficiarios, son personas de extrema pobreza, niños, madres gestantes, lactantes y ancianos.

Ofrece apoyo de salud mediante un centro de salud a un precio simbólico, dando atención en servicios de pediatría, medicina general, odontología, laboratorio clínico y otros servicios.

Finalmente, se subvenciona un centro de rehabilitación para fármaco dependientes, alcohol y ludopatía a personas de cualquier edad en forma ambulatoria.

\section{PROBLEMA GENERAL}

\section{Formulación del Problema}

\section{Problema general}

¿En qué medida el problema de liquidez y rentabilidad con enfoque social influye en la gestión administrativa de la Sociedad de Beneficencia Pública del Callao - Perú 2012-2014?

\section{Problemas Específicos}

1. ¿De qué manera el nivel de razón corriente influye en el cumplimiento de metas y objetivos de la gestión de la Sociedad de Beneficencia Pública del Callao?

2. ¿En qué medida el nivel de capital de trabajo influye en la eficiencia y eficacia de la gestión de la Sociedad de Beneficencia Pública del Callao?

3. ¿En qué medida el nivel de gastos de administración influye en el nivel de competitividad en la gestión de la Sociedad de Beneficencia Pública del Callao?

4. ¿De qué manera el nivel de margen neto de utilidad influye en la optimización de los recursos en la beneficencia pública del Callao?

\section{Justificación teórica}

La realización de la investigación ha proporcionado el conocimiento de las causas de realidad problemática de la Sociedad de Beneficencia Pública del Callao - SBPC, en relación a su rentabilidad y el problema de liquidez, que no permiten que la entidad pueda cumplir efectivamente con sus metas y objetivos, de acuerdo a la misión y visión que el director tiene aprobado, en consecuencia creemos que se justifica en la medida que es necesario cumplir con la población más necesitada, sin que ello signifique el no cumplir con el normal funcionamiento administrativo y operativo en la SBPC, para la consecución de sus objetivos. 


\section{Justificación practica}

El conocimiento de las causas que provocan el problema de la rentabilidad y la liquidez de la SBPC, da las bases para la superación del problema y así se de la posibilidad de desarrollar estrategias, para lograr hacer sostenible y sustentable la economía de la SBPC, lo cual permitirá plantear estrategias adecuadas con la finalidad de maximizar el mejoramiento de la recaudación sin generar dificultades a la población.

\section{Objetivos de la investigación}

\section{Objetivo General}

Determinar si el problema de liquidez y rentabilidad con enfoque social influye en la gestión administrativa de la Beneficencia Pública del Callao - Perú 2012-2014.

\section{Objetivos específicos}

1. Evaluar si la razón corriente influye en el cumplimiento de metas y objetivos de la gestión de la Sociedad de Beneficencia Pública del Callao.

2. Analizar si el nivel de capital de trabajo influye en la eficiencia y eficacia de la gestión de la Beneficencia Pública del Callao.

3. Determinar de qué manera el nivel de gastos de administración influye en el nivel de competitividad en la gestión de la Sociedad de Beneficencia Pública del Callao.

4. Determinar como el margen neto de utilidad influye en la optimización de los recursos de la Beneficencia Pública del Callao

\section{BASES TEÓRICAS}

\section{Liquidez y Rentabilidad}

Liquidez (Liquidity) Pasivos financieros u obligaciones monetarias de las instituciones financieras con el sector privado de la economía. La liquidez puede estar constituida en moneda nacional o moneda extranjera. La liquidez en moneda nacional se divide en:

Dinero: Corresponde a la suma del circulante y los depósitos a la vista mantenidos por el sector privado.
Cuasidinero: Constituido por los depósitos de ahorro, depósitos a plazo, cédulas hipotecarias, letras hipotecarias, bonos emitidos por las instituciones financieras y otros valores. La liquidez en moneda extranjera comprende los depósitos (vista, ahorro y plazo), certificados bancarios en moneda extranjera, bonos, valores emitidos por el Banco Central de Reserva del Perú (Certificados de Divisas, de Libre Disponibilidad y de Plata, bonos) y otras obligaciones en moneda extranjera. Su concepto expresa la facilidad con que un bien o activo puede ser convertido en dinero. El bien líquido por excelencia es el propio dinero, en función del cual se mide la iliquidez de los demás bienes, dependiendo ésta, de la prontitud o facilidad en que cada caso se pueda hacer dicha transformación. Se entiende por liquidez también la disponibilidad inmediata de dinero de un banco, y mide la capacidad de una institución financiera del exterior para hacer frente a sus obligaciones de corto plazo ${ }^{1}$.

\section{¿Qué es la liquidez?}

Según David Wong ${ }^{2}$ Si tuviese la oportunidad de invertir en un proyecto muy seguro y rentable, aunque con una alta probabilidad de que el mismo no pueda afrontar sus obligaciones a corto plazo, ¿invertiría en el proyecto? Probablemente no; o recurriría al mercado de capitales en búsqueda de financiamiento para afrontar el problema. Pero cabe mencionar que en el Perú las fuentes de financiamiento son escasas. Mientras el mercado de capitales no esté desarrollado, la liquidez será el criterio prioritario en las finanzas, inclusive con mayor peso que el riesgo y la rentabilidad. Un ejemplo claro de esta situación son los inversionistas en la Bolsa de Valores de Lima. Muchos invierten sólo en acciones muy líquidas y desdeñan otras que aunque son seguras y rentables, no pueden ser transadas fácilmente. La capacidad de pago a corto plazo no debe entenderse como un concepto absoluto, no existen los términos de activo líquido o ilíquido. El verdadero concepto más bien tiene que ver con una cuestión de grado. Por ejemplo, las cuentas por cobrar son activos más líquidos que los inventarios, pero menos líquidos que la caja. 


\section{RENTABILIDAD}

\section{Indicadores de Rentabilidad}

Existen diversas definiciones y opiniones relacionadas con el término rentabilidad, por ejemplo, Gitman (1997) dice que rentabilidad es la relación entre ingresos y costos generados por el uso de los activos de la empresa en actividades productivas. La rentabilidad de una empresa puede ser evaluada en referencia a las ventas, a los activos, al capital o al valor accionario.

Por otra parte, Aguirre et al. (1997) consideran la rentabilidad como un objetivo económico a corto plazo que las empresas deben alcanzar, relacionado con la obtención de un beneficio necesario para el buen desarrollo de la empresa.

Para Sánchez (2002) la rentabilidad es una noción que se aplica a toda acción económica en la que se movilizan medios materiales, humanos y financieros con el fin de obtener ciertos resultados. En la literatura económica, aunque el término se utiliza de forma muy variada y son muchas las aproximaciones doctrinales que inciden en una u otra faceta de la misma, en sentido general se denomina rentabilidad a la medida del rendimiento que en un determinado periodo de tiempo producen los capitales utilizados en el mismo.

Una actividad es rentable socialmente cuando provee de más beneficios que pérdidas a la sociedad en general, independientemente de si es rentable económicamente para su promotor. Se utiliza como contrapartida al concepto de rentabilidad económica, donde la rentabilidad solo concierne al promotor.

Un ejemplo típico de cálculo de rentabilidad social es el de las líneas de ferrocarril. Una línea es rentable económicamente si los ingresos que obtiene a través de la venta de billetes es mayor que los gastos, mientras que es rentable socialmente si lo que la sociedad ahorra con esa línea (el coste del desplazamiento en coches particulares $\mathrm{u}$ otros medios, el menor tiempo dedicado al transporte, etc.) es mayor que los gastos que genera la infraestructura.

\section{ADMINISTRACIÓN FINANCIERA EN EL ESTADO}

Casas Tragodara (2012), catedrático de la Universidad del Pacífico, señala que la gestión financiera del Estado comprende dos grandes aspectos: primero, la recaudación de ingresos; y segundo, el gasto de dichos recursos en pago de los servicios públicos y en la inversión en infraestructura. Cada uno de estos aspectos está asignado a determinadas instituciones. La recaudación de los ingresos a la Superintendencia Nacional de Aduanas y Administración Tributaria (SUNAT) y el gasto a los ministerios, gobiernos regionales, gobiernos locales, entre otros.

El gasto público se realiza a través de diversos procesos y en diversos niveles, culminando en la eficiencia operativa, tema de la presente tesis, tal como se observa en la Tabla $\mathrm{N}^{\circ} 01$.

Tabla No 01 :

Elementos básicos de la gestión del gasto público: Análisis en tres niveles

\begin{tabular}{|c|l|}
\hline La disciplina fiscal agregada & $\begin{array}{l}\text { Las cifras agregadas del presupuesto deben ser resultado de decisiones expli- } \\
\text { citas que se hacen cumplir y no se limitan a acomodar las tendencias inercia- } \\
\text { les y las demandas de gasto. Los techos agregados para los totales deberían } \\
\text { establecerse antes de adoptar las diferentes decisiones presupuestarias y } \\
\text { deben ser sostenibles en el mediano plazo. }\end{array}$ \\
\hline La asignación a las prioridades & $\begin{array}{l}\text { Las asignaciones presupuestarias se deben basar en las prioridades públicas y } \\
\text { en la eficiencia de los programas públicos. El sistema presupuestario debería } \\
\text { desplazar recursos desde las prioridades menores hacia las más altas y desde } \\
\text { los programas menos eficaces hacia los más eficaces. }\end{array}$ \\
\hline La eficiencia operativa & $\begin{array}{l}\text { Los organismos de línea deberían producir bienes y servicios a un costo } \\
\text { que genere mejoras en la eficiencia y que sea competitivo con los precios de } \\
\text { mercado. }\end{array}$ \\
\hline
\end{tabular}

Fuente: Banco Interamericano de Desarrollo, en Creación de espacio fiscal para reducir la pobreza (2006: 88).

86/ QVIPURAMAYoC | Vol. 24(46) 2016 


\section{GESTIÓN}

Gestión: Viene del latín GESTIO-GESTIONIS que significa ejecutar, lograr un éxito con medios adecuados (Corominas, 1995).

"La acción y efecto de realizar tareas con cuidado, esfuerzo y eficacia- que conduzcan a una finalidad" (Heredia, 1985)

"Actividad profesional tendiente a establecerlos objetivos y medios de su realización, a precisar la organización de sistemas, a elaborar la estrategia del desarrollo y a ejecutar la gestión del personal" (Rementeria, 2008).

"Un conjunto de reglas y métodos para llevar a cabo con la mayor eficacia un negocio o actividad empresarial” (Espasa Calpe, 2008)

\section{METODOLOGÍA}

La presente investigación de acuerdo a la naturaleza del problema planteado y sus objetivos determinados lo identificamos como una investigación de tipo aplicada, por cuanto se utiliza conocimiento pre existente para un propósito concreto y porque nos permitió manipular las operaciones e información obtenida de la entidad en estudio para modificar la realidad con el propósito de analizar el fenómeno mismo que hemos indagado, que es la creación de valor, para su observación y confirmar nuestra hipótesis.

El diseño de la investigación es descriptivo y explicativo, puesto que analiza la problemática que se presenta en la Sociedad de Beneficencia Pública del Callao.

\section{RESULTADOS}

1. ¿Considera Usted que el nivel de razón corriente como indicador de la liquidez, influye en la gestión de la Sociedad de Beneficencia Pública del Callao?

\section{INTERPRETACIÓN:}

Como resultado de la información obtenida, conforme se presenta en la Tabla $\mathrm{N}^{\circ} 02$, nos muestra que el $91.60 \%$ de los encuestados, respondieron que el nivel de razón corriente como indicador de la liquidez, influye en la gestión de la sociedad de beneficencia pública del Callao; sin embargo, un $0.76 \%$ contesto que el nivel de razón corriente como indicador de la liquidez, no influye en la gestión de la sociedad de beneficencia pública del Callao, y el 7.64\% manifestaron desconocer; sumando así el 100\% de la muestra.

Analizando la información que se muestra en el párrafo anterior, podemos manifestar que el mayor porcentaje de los encuestados respondieron por la primera alternativa, lo cual nos demuestra que la mayoría de directivos, funcionarios y servidores consideran que el nivel de razón corriente como indicador de la liquidez, influye en la gestión de la sociedad de beneficencia pública del Callao, lo cual confirma la hipótesis propuesta en relación a la situación de la problemática planteada.

Tabla No 02

Nivel de razón corriente influye en la gestión

\begin{tabular}{|c|c|c|c|}
\hline $\mathrm{N}^{\circ}$ & ALTERNATIVAS & fi & $\%$ \\
\hline 1 & SI & 120 & 91.6 \\
\hline 2 & $\mathrm{NO}$ & 1 & 0.76 \\
\hline 3 & DESCONOCE & 10 & 7.64 \\
\hline \multicolumn{2}{|r|}{ TOTAL } & 131 & $100 \%$ \\
\hline
\end{tabular}

Fuente: Elaboración Propia.

2. ¿En su opinión el nivel de capital de trabajo como indicador de liquidez influye en la gestión de la Sociedad de Beneficencia Pública ubicada en la Provincia Constitucional del Callao?

\section{INTERPRETACIÓN:}

Referente a los alcances de la interrogante, la información recopilada y detallada en la $\mathrm{Tabla} \mathrm{N}^{\circ} 03$, en relación a la pregunta formulada, nos demuestra con claridad que el $90.84 \%$ de los encuestados, respondieron por la primera alternativa o sea consideran que el nivel de capital de capital como indicador de liquidez influye en la gestión de la Sociedad de Beneficencia Publica ubicada en la Provincia Constitucional del 
Callao sin embargo un $1.54 \%$ contestaron marcando por la segunda alternativa, o sea consideran que el nivel de capital de capital como indicador de liquidez no influyen en la gestión de la Sociedad de Beneficencia Pública ubicada en la Provincia Constitucional del Callao y el $7.63 \%$ manifestaron desconocer, sumando así el $100 \%$ de la muestra seleccionada.

Al interpretar los datos e información mostrados en el cuadro anterior podemos apreciar que la gran mayoría de los encuestados, reconocieron que los problemas de liquidez y rentabilidad mostrados por medio de los indicadores si influye en la gestión de la Sociedad de Beneficencia Pública del Callao.

Tabla No 03

Nivel de capital de trabajo influye en la gestión

\begin{tabular}{|c|c|c|c|}
\hline $\mathrm{N}^{\circ}$ & ALTERNATIVAS & fi & $\%$ \\
\hline 1 & SI & 119 & 90.84 \\
\hline 2 & $\mathrm{NO}$ & 2 & 1.53 \\
\hline 3 & DESCONOCE & 10 & 7.63 \\
\hline \multicolumn{2}{|r|}{ TOTAL } & 131 & $100 \%$ \\
\hline
\end{tabular}

Fuente: Elaboración Propia.

3. ¿Considera Usted que el nivel de gastos de administración como indicador de rentabilidad influye en la gestión de la Sociedad de Beneficencia Publica ubicada en la provincia Constitucional del Callao?

\section{INTERPRETACIÓN:}

Como resultado de la información que se presenta en el Tabla $\mathrm{N}^{\circ} 04$ se puede manifestar que el $85.50 \%$ de los encuestados, respondieron por la primera alternativa o sea con un sí, lo cual quiere decir que consideran que el nivel de gastos de administración como indicador de rentabilidad influye en la gestión de la Sociedad de Beneficencia Pública ubicada en la provincia Constitucional del Callao, sin embargo un $6.11 \%$ respondió por la segunda alternativa o sea consideran que el nivel de gastos de administración como indicador de rentabilidad no influye en la gestión de la Sociedad de Beneficencia Pública ubicada en la provincia Constitucional del
Callao, y un $8.39 \%$ contesto por la tercera alternativa sumando el $100 \%$ de la muestra entrevistada.

Buscando una explicación en relación a lo descrito en el párrafo anterior, podemos decir que encontramos con mucha claridad que la gran mayoría de quienes contestaron a las preguntas, reconocieron que los gastos de administración como indicador de rentabilidad influyen en la gestión de la Sociedad de Beneficencia Pública ubicada en la provincia Constitucional del Callao.

\section{Tabla No 04}

Gastos de administración influye en la gestión

\begin{tabular}{|c|c|c|c|}
\hline $\mathrm{N}^{\circ}$ & ALTERNATIVAS & fi & $\%$ \\
\hline 1 & SI & 112 & 85.5 \\
\hline 2 & $\mathrm{NO}$ & 8 & 6.11 \\
\hline 3 & DESCONOCE & 11 & 8.39 \\
\hline \multicolumn{2}{|r|}{ TOTAL } & 131 & $100 \%$ \\
\hline
\end{tabular}

Fuente: Elaboración Propia.

\section{CONCLUSIONES}

1. Los datos e información obtenida como producto de la investigación, permitió establecer que el nivel de razón corriente influye en el cumplimiento de metas y objetivos de la gestión financiera de la Sociedad de Beneficencia Pública del Callao, en el proceso de cumplimiento de sus metas y objetivos.

2. Los datos puestos a prueba nos permitieron establecer que el nivel de capital de trabajo influye positivamente en la eficiencia y eficacia de la gestión administrativa de la Sociedad de Beneficencia Pública del Callao, considerando que permitirá contar con los recursos necesarios e indispensables para el cumplimiento de presupuesto anual.

3. Los datos e información obtenida permitieron precisar a través de la prueba de hipótesis respectiva, que los gastos de administración influyen en el nivel de competitividad en la gestión de la Sociedad de Beneficencia Pública del Callao. 
4. El análisis de los datos e información obtenida permitieron establecer que el nivel del margen neto de utilidad influye positivamente, en la optimización de los recursos administrativos en la Sociedad de Beneficencia Pública del Callao, de tal manera que permitirá contar con los recursos suficientes para el cumplimiento de sus metas y objetivos.

5. En conclusión se ha determinado que los problemas de liquidez y rentabilidad con un enfoque social, influyen en la gestión administrativa de la Sociedad de Beneficencia Pública del Callao - Perú 2012-2014, por cuanto si no se cuenta con los recursos económicos y financieros, será imposible que la SBPC cumpla con sus planes previstos en cada periodo presupuestal.

\section{REFERENCIAS BIBLIOGRÁFICAS}

1. Bunge, M. (1989). Ciencia y Desarrollo. Argentina: Siglo XX.

2. BANCO CENTRAL DE RESERVA DEL PERÚ Glosario de Términos Económicos (2011)

3. Drucker, P. (2003). La Sociedad Post Capitalista. Colombia: Editorial Sudamericana.

4. David Wong (1995) Finanzas en el Perú: Un Enfoque de Liquidez, Rentabilidad y Riesgo 2da Edición 1995 Universidad del Pacifico Lima Perú

5. García, E. (2006). Balanced Scorecard en una Cooperativa de Ahorro y Crédito Hernández R, Fernández C, \& Baptista, P.. (2003). Metodología de la Investigación. México: Mc Graw Hill.

6. Pérez, R. (2007). El Baquijanno Ayer y Hoy. Perú: Julio Toledo Daneri.

7. Toffler, A. (1998). El Shock del futuro. España: Plaza \& Janes.

8. Toffler, A. (1981). La Tercera Ola. Bogotá: Plaza \& Janes.

9. Toffler, A. (1994). El Cambio Del Poder. Barcelona: Plaza \& Janes.

10.Sánchez, F. (2005). Diccionario Contable Financiero, Bursatily de áreas afines, Peru: World Wide International SAC.

11.Sánchez, F. (2005). Diccionario Contable Financiero, Bursátily de áreas afines, Perú: World Wide International SAC.

12.Walton, M. (2004) El Método Deming en la Práctica -6 compañías de éxito que usan los principios del control total de calidad del mundialmente famoso W.E. Deming. Colombia. Grupo Editorial Norma. 